\title{
Eficiência e impacto do contexto na gestão através do DEA: o caso da UEG
}

\author{
Carlos Rosano-Peña ${ }^{\text {a* }}$ \\ a*crosano@unb.br, UNB, Brasil
}

\section{Resumo}

Este trabalho mostra a contribuição do método Análise envoltória de dados (DEA) na avaliação da eficiência técnica de organizações que operam em contextos diferenciados. A aplicação do DEA na Universidade Estadual de Goiás (UEG) demonstra que 20 das 39 unidades analisadas são ineficientes. As 20 sofrem o efeito negativo do entorno medido pelo índice de Desenvolvimento Humano dos Municípios de 2000 - IDH-M. 1solando-se esse efeito, cai o número de ineficientes para 11 e a média dos índices de eficiência técnica passa para 0,95 , o que indica que essas unidades poderiam produzir o mesmo reduzindo os insumos em 5\%, no mínimo. Alternativamente, a UEG, mantendo o nível atual de insumos, poderia incrementar sua produção em 7\%. A pesquisa também mostrou que das 20 unidades pesquisadas 11 estão subdimensionadas e 9 superdimensionadas. Essa informação poderia ser utilizada para reduzir custos e otimizar o porte dessas unidades.

Palavras-chave

Análise envoltória de dados. Avaliação institucional. Contexto. Eficiência.

\section{Introdução}

A eficiência é um dos mais importantes indicadores da qualidade de qualquer sistema. A máxima sinergia dos elementos do sistema faz com que ele possa cumprir sua finalidade com eficiência; já sua entropia (medida da variação ou desordem de um sistema) implica falhas no funcionamento, desarmonia e, portanto, ineficiência. Todavia, a avaliação da eficiência de sistemas complexos não é trivial. Seu êxito depende do nível de complexidade dos processos internos, do relacionamento com o ambiente externo e do conteúdo implícito em cada um dos elementos envolvidos.

Um caso típico de sistema complexo é a educação superior pública. Isto se deve ao dinamismo da ciência, à multiplicidade das funções universitárias, das áreas de conhecimento, dos projetos e cursos oferecidos, dos recursos humanos e patrimoniais envolvidos e à heterogeneidade das regiões e do contexto social em que cada instituição atua. Essa complexidade explica a existência de vários enfoques e a ausência de um modelo conclusivo de avaliação institucional.

Com o intuito de colaborar com a discussão sobre a avaliação universitária, este trabalho tem por objetivo mostrar um método de avaliação da eficiência de unidades produtivas que contemple o contexto socioeconômico diferenciado nos quais essas unidades operam. lsto será realizado por meio de um estudo de caso: a Universidade Estadual de Goiás (UEG) - instituição com muitas unidades distribuídas por diversos municípios do estado.

0 método utilizado, a Análise envoltória de dados - DEA, é uma técnica para apoio à decisão de natureza multicritério que possibilita modelar a complexidade de unidades produtivas. Pode ser empregado para avaliar a eficiência técnica de unidades (chamadas Decision Making Units - DMUs) que empregam múltiplos insumos para produzir diversos bens e/ou serviços, evitando restringi-los a um único produto agregado monetariamente. 0 método compara os insumos e produtos de cada unidade e determina seus índices de eficiência relativa, permitindo assim definir as melhores práticas e as mudanças necessárias para que as unidades ineficientes se tornem eficientes. Adicionalmente, o DEA apresenta inúmeras soluções que possibilitam aperfeiçoar a eficiência das unidades 
analisadas, inclusive descontando o impacto dos fatores não-controláveis. Além disso, pode ser utilizado na identificação de ociosidades e folgas e na formulação de políticas de redução de custos, associada a uma expansão que otimize o potencial de crescimento e o porte ideal das unidades. Portanto, é uma valiosa ferramenta para a avaliação institucional que possibilita orientar e induzir a discussão da gestão num contínuo processo de aprimoramento.

Isto significa que a UEG, com ajuda desse método e seus resultados, poderá fazer um feedback e debater a otimização de seu principal problema econômico: o conflito entre as múltiplas necessidades e a insuficiência dos recursos disponíveis. Conseguirá mostrar melhor sua responsabilidade social e definir a necessidade ou não de recursos adicionais para implementar novos projetos e programas eficientemente, atendendo a demanda reprimida por ensino superior gratuito. Isso não é pouco se se considera que a UEG é uma das maiores universidades brasileiras em número de alunos, com 22\% dos universitários de Goiás.

\section{Referencial teórico}

A primeira definição formal de eficiência deve-se a Koopmans (1951), e o primeiro método de sua quantificação é creditado a Debreu (1951) e a Farrell (1957).

Um processo de produção é eficiente quando emprega a menor quantidade possível de insumos para produzir um número dado de produtos, ou quando obtém o maior nível de produção possível com um determinado nível de insumo. $0 u$, ainda, no dizer de Koopmans (1951, p. 60):

[...] um gestor, que produz dois ou mais produtos com certos insumos, é eficiente se ele somente conseguir aumentar a produção de um bem, diminuindo a produção de algum outro, ou quando é tecnologicamente impossivel reduzir algum insumo sem simultaneamente incrementar algum outro recurso para manter o mesmo nivel de produção.

Entre as técnicas mais utilizadas para quantificar a eficiência encontram-se os métodos paramétrico e não paramétrico. 0 primeiro é mais tradicional. A eficiência é avaliada a partir de uma relação funcional concreta entre os inputs utilizados e a máxima quantidade de outputs produzidos, estimando-se os parâmetros dessa função produção por meio de técnicas econométricas. 0 trabalho pioneiro de Coleman et al. (1966) é um exemplo da utilização desse método na educação. Outra publicação clássica corresponde a Hanushek (1986), que analisou 147 estimativas da função de produção educacional.
0 não paramétrico assume hipóteses mais flexíveis sobre o comportamento (distribuição) das variáveis e não requer a especificação a priori de nenhuma relação funcional entre insumos e produtos, ficando livre dos possíveis erros dessa especificação. Nesse método, o conjunto de possibilidade de produção (CPP) é determinado de uma forma mais indutiva, por meio do conjunto de todos os processos produtivos tecnologicamente factíveis, estimado a partir das práticas empiricamente observadas. Constrói-se a fronteira do CPP por meio da combinação linear das observações eficientes e mensura-se a eficiência a partir da distância que separa as unidades dessa fronteira. Portanto, ele dispensa a tradicional hipótese paramétrica de comportamento eficiente das unidades analisadas, muito importante na educação pública, na qual não existe o mecanismo de liquidação automática que expulsa as unidades ineficientes, como acontece com o mecanismo de falência da empresa privada. Além disso, o método não paramétrico possibilita o estudo de situações de múltiplos outputs/inputs e não usa o preço monetário como ponderador. lsto aumenta a confiabilidade do modelo, uma vez que os preços, afetando e sendo afetados por inputs e outputs, são ponderadores instáveis no tempo e no espaço e incorporam as possíveis implicações da ineficiência técnica. Essa é outra das principais razões da popularidade desse método no estudo da educação pública - setor típico na produção de múltiplos bens e serviços na ausência ou escassa presença de mercado. Seu desenvolvimento foi produto da proposta de utilização do índice de eficiência feita por Farrell (1957) em um estudo no setor educacional e é atribuído a Charnes, Cooper e Rhodes (1978), que deram a conhecer o método como Data Envelopment Analysis - DEA.

0 método DEA vem apresentando um grande sucesso no estudo da eficiência de instituições de ensino e de outros setores, especialmente no de organizações públicas e sem fins lucrativos. Uma importante compilação bibliográfica sobre o DEA e suas aplicações empíricas vem sendo atualizada na página web http://www.deazone.com. 0 registro de publicações sobre o DEA na educação nesse site já era de 65 em 21/3/2010.

0 modelo inicial proposto por Charnes, Cooper e Rhodes (1978), designado por CCR, foi desenhado para uma análise com retornos constantes de escala - RCE, na qual qualquer variação nos inputs produz uma variação proporcional nos outputs. Posteriormente foi estendido por Banker, Charnes e Cooper (1984) para incluir retornos variáveis (não proporcionais) de escala - RVE e passou a chamar-se BCC. Cada um desses modelos pode ser desenhado sob duas formas básicas de maximizar a eficiência: 1) reduzir o consumo 
de insumos mantendo o nível de produção, ou seja, orientado ao input $(10)$; 2) aumentar a produção conservando os níveis de consumo dos insumos, ou seja, orientado ao output (00).

0 modelo CCR-10 parte da definição relativa de produtividade total dos fatores (PTF), entendida como a soma ponderada dos produtos dividida pela soma ponderada dos insumos, sendo seus pesos determinados por um problema de maximização sujeito à restrição de que todos os quocientes de PTF sejam menores ou iguais a um. Geometricamente a ideia, no caso mais simples (um input e um output), é construir uma reta que partindo da origem determine uma combinação linear com as melhores práticas com RCE. Essa reta é a fronteira eficiente ou produto potencial e em relação a ela a ineficiência das outras unidades pode ser avaliada. Essa superfície eficiente é um plano em $\mathrm{R}^{3}$ quando estão envolvidas três variáveis, ou um hiperplano para um vetor output-input de dimensão maior que três.

A formulação (1) mostra o modelo inicial do DEA com orientação aos insumos (CCR-OI) para uma unidade $o$ qualquer (de um grupo de $\mathrm{N}$ organizações homogêneas) que produz o vetor $y_{r o}$, utilizando o vetor insumo $x_{i o}$ e uma tecnologia com retorno constante de escala (RCE).

$$
\operatorname{Max} h_{o}=\left[\sum_{r=1}^{m} u_{r} y_{r o}\right] \div\left[\sum_{i=1}^{n} v_{i} x_{i o}\right]
$$

Sujeito a: $\left[\sum_{r=1}^{m} u_{r} y_{r j}\right] \div\left[\sum_{i=1}^{n} v_{i} x_{i j}\right] \leq 1$; $u_{r}, v_{i} \geq 0 ; j=1, \ldots, N ; r=1, \ldots, m ; i=1, \ldots, n$.

A formulação (1) é transformada em um problema de programação linear (PPL), para evitar infinitas soluções, e pode ser condicionada a que $u_{r}$ e $v_{i}$ nunca fiquem abaixo de um valor muito pequeno não arquimediano $\varepsilon$ (onde $\varepsilon$ pode ser igual a $10^{-6}$ ), para impedir que os pesos cheguem a zero; ou seja, para evitar que algum produto ou insumo seja marginalizado da determinação da eficiência. A nova formulação CCR-M-O1, conhecida como forma multiplicativa, é dada por (2) e sua equivalente, orientada aos outputs (CCR-M-00), por (3).

$$
\operatorname{Max} h_{o}=\sum_{r=1}^{m} u_{r} y_{r o}
$$

Sujeito a:

$$
\begin{aligned}
& \sum_{i=1}^{n} v_{i} x_{i o}=1 ; \sum_{r=1}^{m} u_{r} y_{r j} \leq \sum_{i=1}^{n} v_{i} x_{i j} ; \\
& u_{r}, v_{i} \geq \varepsilon ; \\
& j=1, \ldots, N ; r=1, \ldots, m ; i=1, . ., n .
\end{aligned}
$$

$\operatorname{Min} \varphi_{o}=\sum_{i=1}^{n} v_{i} x_{i o}$
Sujeito a:

$$
\begin{aligned}
& \sum_{i=1}^{n} u_{r} y_{r o}=1 ; \sum_{r=1}^{m} u_{r} y_{r j} \geq \sum_{i=1}^{n} v_{i} x_{i j} ; \\
& u_{r}, v_{i} \geq \varepsilon ; \\
& j=1, \ldots, N ; r=1, \ldots, m ; i=1, \ldots, n .
\end{aligned}
$$

A diferença básica entre essas formulações está no fato de que (2) determina a redução máxima necessária dos inputs e (3) encontra o aumento máximo necessário nos outputs para que as DMUs se tornem eficientes. Os dois modelos devem apresentar resultados equivalentes, já que se está trabalhando com modelos que consideram retornos constantes de escala. Apenas as unidades com $h=1 \mathrm{em}$ (2) alcançarão em (3) $\varphi=1$. As DMUs ineficientes apresentarão um $\varphi>1$, que terá um valor inverso ao do modelo (2): $\varphi=1 / h$. Assim, se o $h$ de uma unidade com (2) é 0,8 , em (3) terá um $\varphi=1,25=1 / 0,8$. Sua interpretação indicará que a unidade deverá aumentar de forma proporcional (radial) os outputs em 25\% para ser eficiente. Alternativamente, essa unidade, mantendo o nível atual de produção, poderia diminuir de forma proporcional seus insumos em 20\%.

A medida de eficiência com (2) pode ser representada conforme a Figura 1, que ilustra a fronteira 10, ou seja, as combinações mínimas dos insumos $x 1$ e $x 2$ necessárias para produzir uma unidade de y. Qualquer ponto na fronteira é eficiente e possui $\mathrm{h}=1$. Assim, nenhuma redução nas DMUs C e B é viável para manter o mesmo nível de produção. As unidades que se encontram acima dessa curva são ineficientes. Portanto, $h_{\mathrm{F}}<1$, sendo F' o valor que os insumos deveriam assumir para $\mathrm{F}$ tornar-se eficiente. A diminuição possível dos insumos para as unidades ineficientes é dada pela projeção radial da observação até a fronteira.

Contudo, observa-se que a determinação da fronteira eficiente pelo PPL resulta em segmentos lineares. Isso identifica problema de folgas na

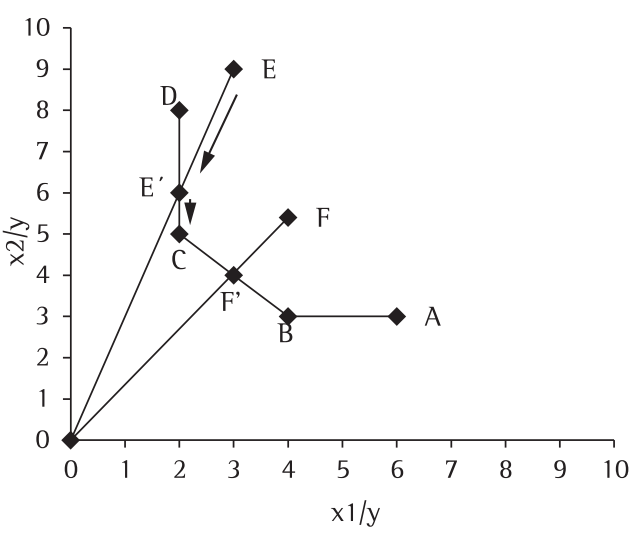

Figura 1. Fronteira eficiente, orientação inputs. 
mensuração da eficiência em razão da ocorrência de trechos da fronteira paralelos aos eixos de insumos e de produtos. Por exemplo, na Figura 1, as DMUs situadas nos trechos paralelos a um dos insumos (D, E' e A) têm um índice de eficiência igual a 1, embora o desempenho dessas unidades possa ser melhorado reduzindo parte desse insumo sem redução de produção.

Para esclarecer essa situação, Cooper, Seiford e Tone (2007, p. 45) formularam a seguinte definição:

[...] uma DMU é eficiente se e somente se seu índice é 1 e todas as folgas (tanto nos insumos, como nos produtos) forem iguais a 0 (zero); caso contrário, a unidade deve ser qualificada como ineficiente.

Essa definição, denominada eficiência de Pareto-Koopmans, é mais restrita que a definição que só considera o índice de eficiência de Farrell. Portanto, segundo a Figura 1, alguns autores chamam a linha formada pelas unidades B e C de fronteira Pareto-Koopmans ou fronteira fortemente eficiente. 0 contorno que passa por BC e inclui tanto o segmento BA como o segmento vertical acima da unidade C é chamado de fronteira Debreu-Farrell eficiente ou fronteira fracamente eficiente (LINS; ANGULO-MEZA, 2000, p. 14-30). Assim, o ponto E deve fazer um movimento radial, projetando-se até o ponto E', considerado eficiente nos conceitos de Debreu e Farrell, pois está localizado na fronteira, mas não no conceito de Koopmans, já que o insumo x2 poderia ser reduzido de E' para C sem mudar o nível de produção. Portanto, D precisa deslocar-se para C, como E'. Esse é chamado de movimento não radial ou melhoria de folga.

0 mesmo raciocínio é válido na identificação da eficiência e das folgas com o modelo (3), no espaço output da curva de possibilidades de produção.

Aqui é necessário observar também que correspondendo a qualquer PPL (primal), sempre existe outro PPL (dual) que é a sua contrapartida. No DEA, o dual apresentará menos restrições, pois se exige que a amostra de DMUs seja maior que o número de variáveis. Portanto, e por ter solução mais simples, o dual tem preferência.

Os resultados do dual são importantes porque fornecem os preços-sombra (eficiência marginal - $\lambda$ ), que determinam, junto com as DMUs referência, os pontos de projeção das unidades ineficientes sobre a fronteira Pareto-Koopmans. Por exemplo, no caso de F na Figura 1, a projeção sobre a fronteira (F') resultará da combinação linear das DMUs eficientes referência (B e C) nas proporções dadas por $\lambda_{\mathrm{B}}$ e $\lambda_{\mathrm{C}}$, obtidos a partir do dual que avalia F, ou seja:

$$
\hat{x}_{i F},=\lambda_{B} x_{i B}+\lambda_{C} x_{i C} ; \hat{y}_{r F}=\lambda_{B} y_{r B}+\lambda_{C} y_{r C},
$$

onde $\left(\hat{x}_{i F}, \hat{y}_{r F}\right)$ são os vetores das metas de melhorias de F.

Além disso, a diferença dos valores da projeção sobre a fronteira Pareto-Koopmans e da redução radial indicará as folgas $\left(\mathrm{s}_{\mathrm{i}}^{+}\right.$e $\left.\mathrm{s}_{\mathrm{r}}^{-}\right)$:

$$
s_{r}^{+}=\sum_{j=1}^{N} y_{i j} \lambda_{j}-\varphi_{j} y_{r j} ; s_{i}^{-}=x_{i j}-\sum_{j=1}^{N} x_{i j} \lambda_{j}
$$

Para o caso anterior, em que existam N DMUs com i inputs e r outputs, o PPL dual associado ao (2) da DMU é (4), denominado CCR-E-10. Observa-se que as variáveis de decisão são $\lambda_{\mathrm{j}}$, sendo, para uma $\mathrm{DMU}_{j}$ eficiente, $\lambda_{\mathrm{j}}=1$ e $\Sigma \lambda_{i}=O(j \neq l)$, e para uma $\mathrm{DMU}_{b}$ ineficiente, $-\lambda_{\mathrm{b}}=0$ e $\Sigma \lambda_{j}>0(j \neq b)$. Assim, a projeção de uma unidade ineficiente qualquer é expressa como uma combinação linear das unidades $\operatorname{com} \lambda>0$.

$$
\begin{aligned}
& \text { Min } h_{o} \text { S.a }: \sum_{j=1}^{N} \lambda_{j} y_{i j} \geq y_{r o} ; \\
& x_{r j} h_{o} \geq \sum_{j=1}^{N} x_{i j} \lambda_{j} ; \\
& \lambda_{j} \geq 0 \text { e } h_{o} \text { - irrestrito }
\end{aligned}
$$

A reformulação dual de (3) é (5) e está determinada pelas mesmas regras que transformaram o PPL primal no dual 10.

As restrições de desigualdades do PPL CCR-E-00 (5) podem ser modificadas por restrições de igualdades com a incorporação das folgas dos inputs e dos outputs $\left(\mathrm{s}_{\mathrm{i}}^{-} \mathrm{e}_{\mathrm{r}}^{+}\right)$. Assim, o modelo (5) pode ser reformulado como (6). Esse último modelo, na realidade, tem duas funções na promoção da eficiência de Pareto-Koopmans: 1) determinar o máximo aumento radial possível que deve produzir-se na DMU analisada; 2) maximizar a soma das folgas inputs e outputs. Por isso ele é chamado por alguns autores de modelo de duas etapas (COLL; BLASCO, 2006, p. 50).

$$
\begin{aligned}
& \operatorname{Max} \varphi_{o} \\
& \text { S.a }: \sum_{j=1}^{N} \lambda_{j} y_{i j} \geq \varphi_{o} y_{r o} ; \\
& \sum_{j=1}^{N} \lambda_{j} x_{i j} \leq x_{i o} ; \quad \lambda_{j} \geq 0 .
\end{aligned}
$$

$$
\begin{aligned}
& \operatorname{Max} \varphi_{o}+\varepsilon\left(\sum_{i=1}^{n} S_{i}^{-}+\sum_{r=1}^{m} S_{r}^{+}\right) \\
& \text {S.a }:\left(\sum_{j=1}^{N} \lambda_{j} y_{i j}\right)-S_{r}^{+}=\varphi_{o} y_{o} ; \\
& \left(\sum_{j=1}^{N} \lambda_{j} x_{i j}\right)+S_{i}^{-}=x_{i o} ; \lambda_{j}, S_{i}^{-}, S_{r}^{+} \geq 0 .
\end{aligned}
$$


Outra adaptação dos modelos DEA ocorreu devido à necessidade de incluir inputs não controláveis e fatores ambientais externos na análise, ou seja, variáveis que intervêm e influenciam o processo produtivo mas não são passíveis de alteração e não estão sob controle dos gestores. Embora essas variáveis sejam de natureza diferente, a linha que as separa é muitas vezes tão difusa que fica a critério do pesquisador sua distinção. Segundo Muñiz (2001, p. 199), poucas vezes os inputs não discrecionais e os fatores ambientais são diferenciados nos trabalhos publicados que usam o DEA e, por isso, neste trabalho, eles serão caracterizados indiscriminadamente como variáveis não controláveis ou contexto. Exemplos de inputs não controláveis são as habilidades cognitivas, atitudes, outras características inatas do aluno, que estão fortemente correlacionadas com fatores que formam o entorno externo da escola, no qual entra o comprometimento dos pais com a educação, o ambiente socioeconômico e familiar do aluno. Ambos são quase indissociáveis e embora sejam não controláveis mostram-se em algumas pesquisas mais importantes que os fatores controláveis na determinação do desempenho escolar (COLEMAN et al., 1966).

Os primeiros modelos para lidar com variáveis não controláveis foram propostos por Banker e Morey (1986). São os mais aplicados e simples. Uma adaptação do modelo dual com 10 e 00, considerando a presença de insumos não controláveis, se reescreve como (7) e (8), respectivamente.

\section{$\operatorname{Min} \pi_{o}$}

S.a : $\sum_{j=1}^{N} \lambda_{j} y_{i j} \geq y_{r o}$

$\sum_{j=1}^{N} \lambda_{j} x_{i j} \leq \pi_{o} x_{i o}^{c} ;\left(i \in x^{c}\right)$

$\sum_{j=1}^{N} \lambda_{j} x_{i j}=x_{i o}^{N C} ;\left(i \in x^{N C}\right)$;

$\lambda_{j} \geq 0, \pi_{o}$ irrestrito.

\section{$\operatorname{Max} \rho_{o}$}

S.a: $\sum_{j=1}^{N} \lambda_{j} y_{i j} \geq \rho_{o} y_{r o}$;

$\sum_{j=1}^{N} \lambda_{j} x_{i j} \leq x_{i o}^{C} ;\left(i \in x^{C}\right)$;

$\sum_{j=1}^{N} \lambda_{j} x_{i j}=x_{i o}^{N C} ;\left(i \in x^{N C}\right)$;

$\lambda_{j} \geq 0, \rho_{0}$ irrestrito.

Observa-se que em ambas as orientações os índices estão relacionados apenas às variáveis controláveis $\left(\mathrm{x}^{\mathrm{c}}, \mathrm{y}\right) .0$ objetivo das formulações é identificar as melhoras necessárias nas variáveis controláveis, respeitando a restrição relacionada com o valor fixo das variáveis não controláveis $\left(\mathrm{x}^{\mathrm{NC}}\right)$. Dessa forma, se num PPL tem-se como variável controlável um input, digamos professores, e uma variável que caracterize o contexto como não controlável, o PPL envolvido apresenta uma redução radial apenas no input professor.

A proposta de Banker e Morey, combinada com outros modelos, permite identificar e isolar o impacto das variáveis não controláveis na gestão das unidades analisadas. Esse propósito, ainda, é atingido utilizando-se outros modelos que, inclusive, combinam métodos paramétricos e não paramétricos. Um estudo sobre esses modelos encontra-se no trabalho de Muñiz (2001) e de Angulo-Meza e Santos (2007). No presente trabalho se utilizará uma simples adaptação da metodologia utilizada por Lozano-Vivas, Pastor e Pastor (2002) na análise da eficiência do sistema bancário europeu, como se verá na metodologia.

Conforme já mencionado, os modelos CCR pressupõem que as DMUs operem com retornos constantes de escala. Estudos empíricos confirmam que a maior parte dos setores produtivos encontra-se produzindo com retornos constantes de escala (VASCONCELLOS; OLIVEIRA, 1996, p. 138). Porém, em situações de concorrência imperfeita, principalmente no setor público, existem organizações que podem estar operando com retornos crescentes e decrescentes de escala. Assim, a análise pode ser melhor formulada com os modelos BCC. Esses modelos consideram que um acréscimo nos inputs pode promover um acréscimo no output não necessariamente proporcional. Eles avaliam o conjunto de DMUs dependendo da natureza dos rendimentos de escala que caracteriza o processo produtivo, ou seja, permitem comparar unidades de portes distinto.

Os modelos BCC são estruturalmente similares aos CCR. A diferença entre eles éque, no BCC-M-10, adiciona-se uma constante $\mathrm{k}$ à soma ponderada dos produtos. A variável $\mathrm{k}$ informa se os retornos de escala são constantes, crescentes ou decrescentes para a projeção ótima. Se $k>0$, a produção é caracterizada como retorno de escala crescente; se $k<0$, existe retorno de escala decrescente; e se $\mathrm{k}=0$ diz-se que é com retorno de escala constante. Observa-se em (9) o BCC-M-10 e, em (10), o BCC-E-10 dual.

$$
\begin{aligned}
& \text { Max } \tau_{o}=\sum_{r=1}^{m} u_{r} y_{r o}+k \\
& \text { S.a : } \sum_{i=1}^{n} v_{i} x_{i o}=1 ; \\
& \sum_{r=1}^{m} u_{r} y_{i j}-\sum_{i=1}^{n} v_{i} x_{i j}+k \leq 0 ; \\
& u_{r}, v_{1} \geq 0 ; k-\text { irrestrito. }
\end{aligned}
$$


$\operatorname{Min\tau }_{o}$

S.a: $\sum_{j=1}^{N} \lambda_{j} y_{r j}=y_{r o}$;

$x_{i o} \tau_{o} \leq \sum_{j=1}^{N} x_{i j} \lambda_{j} ; \quad \sum_{j=1}^{N} \lambda_{j}=1 ;$

$\lambda_{j} \geq 0 ; \tau_{o}$-irrestrito.

Desse modo, os índices têm diferentes conotações: a) eficiência com RCE ( $\left.E T_{C R}\right)$, chamada de eficiência global; b) a eficiência com RVE (ET $\left.\mathrm{VR}_{\mathrm{V}}\right)$, conceituada como eficiência pura. Além disso, a relação entre as duas anteriores, definida como eficiência de escala: $\mathrm{EE}=\mathrm{ET}_{\mathrm{CR}} / \mathrm{ET}_{\mathrm{VR}}$.

Caso se pretenda maximizar a produção dados os níveis de insumos, o BCC-M-00 é dado por (11). Seu dual (BCC-E-00) correspondente é (12).

$$
\begin{aligned}
& \text { Max } \theta_{o}=\sum_{i=1}^{n} v_{i} x_{i o}-k \\
& \text { S.a : } \sum_{r=1}^{m} u_{r} y_{r o}=1 ; \\
& \sum_{r=1}^{m} u_{r} y_{r j}-\sum_{i=1}^{n} v_{i} x_{i j}-k \geq 0 ; \\
& u_{r}, v_{1} \geq 0 ; k-\text { irrestrito. }
\end{aligned}
$$

$$
\begin{aligned}
& \text { Min } \theta_{o} \\
& \text { S.a : } \sum_{j=1}^{N} \lambda_{j} y_{r j} \geq \theta_{o} y_{r o} ; \\
& x_{i o} \geq \sum_{j=1}^{N} x_{i j} \lambda_{j} ; \sum_{j=1}^{N} \lambda_{j}=1 ; \\
& \lambda_{j} \geq 0 ; \theta_{o} \text {-irrestrito. }
\end{aligned}
$$

A eficiência pura dos modelos BCC-00 pode ser dada por $1 / \theta$, de tal forma que a DMU será eficiente se $\theta=1$. Mas, a medida de eficiência desses modelos já não é equivalente ao índice dos modelos BCC-Ol: $\tau \neq 1 / \theta$, a menos que a unidade apresente retorno constante de escala.

A seguir são apresentados os modelos duais BCC com variáveis não controláveis e orientação ao input e ao output, (13) e (14), respectivamente.

$\operatorname{Min} \eta_{o}$

$$
\begin{aligned}
& \text { S.a }: \sum_{j=1}^{N} \lambda_{j} y_{r j} \geq y_{r o} ; \\
& \sum_{j=1}^{N} \lambda_{j} x_{i j} \leq \eta_{o} x_{i o}^{C} ;\left(i \in x^{C}\right) ; \\
& \sum_{j=1}^{N} \lambda_{j} x_{i j}=x_{i o}^{N C} ;\left(i \in x^{N C}\right) ; \\
& \sum_{j=1}^{N} \lambda_{j}=1 ; \lambda_{j} \geq 0, \eta_{o}-\text { irrestrito. }
\end{aligned}
$$

$\operatorname{Max} \omega_{o}$

$$
\begin{aligned}
& \text { S.a : } \sum_{j=1}^{N} \lambda_{j} y_{r j} \geq \omega_{o} y_{r o} ; \\
& \sum_{j=1}^{N} \lambda_{j} X_{i j} \leq x_{i o}^{C} ;\left(i \in x^{C}\right) ; \\
& \sum_{j=1}^{N} \lambda_{j} X_{i j}=x_{i o}^{N C} ;\left(i \in x^{N C}\right) ;
\end{aligned}
$$

$\sum_{j=1}^{N} \lambda_{j}=1 ; \lambda_{j} \geq 0, \omega_{o}$-irrestrito.

\section{Metodologia da pesquisa}

$\mathrm{Na}$ avaliação dos serviços da UEG, que possui unidades de diversos portes em contextos socioeconômicos diferenciados, os modelos mais adequados são aqueles que consideram os retornos variáveis de escala e contemplam as variáveis não controláveis. Assim, optou-se por um procedimento baseado nos modelos BCC e de Banker-Morey e pela metodologia utilizada por Lozano-Vivas, Pastor e Pastor (2002).

Na primeira etapa desse procedimento, avalia-se a eficiência, considerando-se apenas as variáveis controláveis conforme BCC-E-10 (10); ou seja, as DMUs serão comparadas sem considerar a possibilidade de estarem operando em contextos diferenciados. Isso resulta nos índices $\tau$, que reúnem tanto o desempenho decorrente da gestão individual das DMUs quanto os efeitos imputados ao entorno. Porém, os gestores não podem ser responsabilizados por ineficiências que, em parte, congregam influências negativas não controláveis por eles. Para fazer uma avaliação justa e isolar esse efeito, numa segunda etapa se incorpora ao modelo uma variável não controlável (entorno), através do modelo BCC-10 (13). Esse modelo forma uma nova fronteira eficiente com os menores valores da nova variável entorno e determina os índices $\eta$, que devem ser maiores ou iguais a $\tau$, já que as unidades que operam em contextos desfavoráveis podem ter uma melhora em sua eficiência $\eta$. É conhecido que o acréscimo de uma variável no PPL pode melhorar o valor da função objetivo. Essa melhora exprime a distância existente entre as duas fronteiras ou a diferença entre as duas medidas [(10) e (13)]. Determina o coeficiente $\alpha=\eta / \tau$ (onde $0<\alpha \leq 1$ ), que capta o possível impacto negativo do entorno e pode ser interpretado como a parte da ineficiência presente em (10) decorrente do impacto ambiental. Quanto menor $\alpha$, maior o efeito negativo. Quando $\alpha=1, \tau=\eta$, o que significa que o impacto negativo é nulo e a ineficiência existente será explicada exclusivamente pela gestão dos recursos controláveis. Assim, a primeira e segunda etapas do procedimento proposto permitem avaliar a eficiência total e a eficiência de gestão interna das unidades, respectivamente. 
A principal vantagem desse procedimento é sua simplicidade. No entanto, ele apresenta uma inconveniência: só corrige os índices das DMUs que são afetadas negativamente pelo ambiente.

Em seguida, utilizam-se BCC-10 (13) e BCC-00 (14), que permitem definir as melhores práticas, as unidades ineficientes e as mudanças necessárias para que as ineficientes (e a UEG como um todo) se tornem eficientes. Por último, com ajuda dos modelos (8) e (14), se discriminará a eficiência global entre eficiência pura e de escala.

Para a aplicação do procedimento proposto, consideraram-se as 39 DMUs mais importantes segundo o Relatório de gestão da UEG 2007. Não foram incluídos os 12 polos, as três extensões e a unidade de Aparecida de Goiânia.

Quanto às variáveis que devem ser selecionadas para a avaliação de serviços educacionais, existe um debate aberto. Segundo Palomares-Montero, García-Aracil e Castro-Martínez (2008), que fazem uma revisão bibliográfica sobre os indicadores de desempenho das universidades, até hoje não há um consenso, devido à complexidade da prestação de serviços educacionais. Na grande maioria das publicações, ta seleção de variáveis é feita segundo a opinião de algum especialista ou a disponibilidade de dados. Neste trabalho, fez-se a escolha pela base dos dados fornecida pela diretoria de planejamento da UEG. Esses dados permitiram discriminar insumos e produtos considerando a atuação universitária como qualquer outra ação de natureza produtiva, representada pela relação técnica entre um conjunto de fatores produtivos (inputs) que se combinam adequadamente para obter certos outputs.

Os insumos selecionados são três: número de funcionários - $\left(1_{1}\right)$, número de professores $-\left(1_{2}\right)$ e área construída em $\mathrm{m}^{2}-\left(\mathrm{l}_{3}\right)$. As variáveis $\mathrm{l}_{1}$ e $\mathrm{l}_{2}$ representam 80\% das despesas correntes totais da universidade (UNIVERSIDADE..., 2007, p. 93), considerando tanto os efetivos quanto os de contrato temporário. Não se faz distinção entre professores com dedicação exclusiva, 40 horas, ou parcial, bem como quanto à qualificação deles. A área construída $1_{3}$ caracteriza, fundamentalmente, o investimento fixo realizado. Essa variável é de fato um input não controlável no curto prazo, porém, para identificar as potenciais melhoras, foi caracterizada como controlável.

Os produtos são cinco: projetos de pesquisa registrados $\left(\mathrm{O}_{1}\right)$, cursos de graduação $\left(\mathrm{O}_{2}\right)$, cursos superiores de qualificação profissional - tecnólogos, sequenciais e licenciatura plena parcelada $\left(\mathrm{O}_{3}\right)$, cursos de pós-graduação $\left(\mathrm{O}_{4}\right)$ e número de alunos regulares $\left(\mathrm{O}_{5}\right)$. Eles representam a tríade de sustentação do ensino superior: pesquisa, ensino e extensão. A variável $0_{1}$ é um indicador da produção científica; $\mathrm{O}_{2}, \mathrm{O}_{4}$ e $\mathrm{O}_{5}$, do trabalho de ensino; e $\mathrm{O}_{3}$, da extensão.
A outra variável é o contexto. Conforme já enfatizado, subestimar o contexto representaria superestimar a eficiência daquelas DMUs expostas a condições favoráveis em detrimento daquelas menos favorecidas. Geralmente o contexto é caracterizado por um conjunto de elevado número de variáveis não controláveis de natureza individual, econômica, social e cultural que precisa ser simplificado com critérios coerentes utilizando-se a análise fatorial. Dada a indisponibilidade dessa informação, neste trabalho utilizou-se uma variável aproximada (proxy): o Índice de Desenvolvimento Humano dos Municípios - IDH-M de 2000. Ele sintetiza adequadamente o entorno diferenciado das DMUs e está correlacionado com muitos inputs não controláveis. Partiu-se do consenso de que quanto menor o $\mathrm{IDH}-\mathrm{M}$, maior deve ser a importância social da DMU, e, portanto, melhor o índice de eficiência.

$\mathrm{Na}$ pesquisa foram utilizados o software especializado DEA-SAED, para executar o BCC-E-10 (10), e o GAMS, para os modelos com variáveis não controláveis.

\section{Resultados obtidos e análise}

Seguindo o procedimento metodológico exposto, os principais resultados da primeira e segunda etapas são ilustrados na Tabela 1. A segunda coluna, decorrente do modelo BCC-E-10 (10), mostra a eficiência calculada sem considerar o entorno. Observa-se que a média dos índices é 0,90 e que existem 19 DMUs eficientes e 20 ineficientes. Porém as DMUs se encontram em contextos diferenciados, portanto esses índices devem estar subdimensionados, atribuindo aos insumos resultado gerado por outros fatores externos não incluídos na análise. Daí a necessidade de introduzir o entorno. A terceira coluna da Tabela 1 exibe a eficiência de gestão, considerando-se o contexto incontrolável, através de BCC-10 (13). Ressalta-se o aumento do número de DMUs eficientes para 28 e a queda das ineficientes para 11. Ou seja, nove DMUs que passam a ser eficientes com (13) apresentavam em (10) uma ineficiência causada exclusivamente por fatores do contexto. A média dos índices de (13) passa para 0,95 , o que indica que as DMUs ineficientes, depurado o efeito negativo do entorno, poderiam produzir o mesmo reduzindo os insumos em 5\% no mínimo. A quarta coluna apresenta o coeficiente que capta o efeito negativo que o entorno exerce no índice. Observa-se que, das $20 \mathrm{DMUs}$ inicialmente ineficientes, todas foram, de alguma forma, prejudicadas pelo entorno, com $\alpha<1$. A mais afetada é a 11. Assim podemos concluir que a eficiência não é obra única da gestão dos recursos controláveis. As últimas linhas da Tabela 1 mostram que a eficiência poderia melhorar em média 10\%, sendo atribuído ao fator ambiental cinco pontos percentuais e à ineficiência de gestão os outros cinco, demonstrando-se assim a 
Tabela 1. Eficiência total, eficiência de gestão e impacto do contexto.

\begin{tabular}{|c|c|c|c|}
\hline DMU & Score $\tau(10)$ & Score $\eta(13)$ & $\alpha=\tau / \eta$ \\
\hline 1 & 1,000 & 1,000 & 1,000 \\
\hline 2 & 1,000 & 1,000 & 1,000 \\
\hline 3 & 0,897 & 1,000 & 0,897 \\
\hline 4 & 0,763 & 1,000 & 0,763 \\
\hline 5 & 0,968 & 1,000 & 0,968 \\
\hline 6 & 0,723 & 0,952 & 0,759 \\
\hline 7 & 1,000 & 1,000 & 1,000 \\
\hline 8 & 1,000 & 1,000 & 1,000 \\
\hline 9 & 1,000 & 1,000 & 1,000 \\
\hline 10 & 1,000 & 1,000 & 1,000 \\
\hline 11 & 0,537 & 1,000 & 0,537 \\
\hline 12 & 1,000 & 1,000 & 1,000 \\
\hline 13 & 0,621 & 0,650 & 0,955 \\
\hline 14 & 0,873 & 0,901 & 0,969 \\
\hline 15 & 1,000 & 1,000 & 1,000 \\
\hline 16 & 0,862 & 0,936 & 0,921 \\
\hline 17 & 1,000 & 1,000 & 1,000 \\
\hline 18 & 1,000 & 1,000 & 1,000 \\
\hline 19 & 0,654 & 0,777 & 0,842 \\
\hline 20 & 0,833 & 1,000 & 0,833 \\
\hline 21 & 0,709 & 0,750 & 0,946 \\
\hline 22 & 0,777 & 0,778 & 0,999 \\
\hline 23 & 1,000 & 1,000 & 1,000 \\
\hline 24 & 0,893 & 1,000 & 0,893 \\
\hline 25 & 1,000 & 1,000 & 1,000 \\
\hline 26 & 1,000 & 1,000 & 1,000 \\
\hline 27 & 1,000 & 1,000 & 1,000 \\
\hline 28 & 0,948 & 1,000 & 0,948 \\
\hline 29 & 0,861 & 1,000 & 0,861 \\
\hline 30 & 1,000 & 1,000 & 1,000 \\
\hline 31 & 1,000 & 1,000 & 1,000 \\
\hline 32 & 1,000 & 1,000 & 1,000 \\
\hline 33 & 0,893 & 1,000 & 0,893 \\
\hline 34 & 0,932 & 0,936 & 0,996 \\
\hline 35 & 1,000 & 1,000 & 1,000 \\
\hline 36 & 1,000 & 1,000 & 1,000 \\
\hline 37 & 0,765 & 0,769 & 0,995 \\
\hline 38 & 0,976 & 0,998 & 0,978 \\
\hline 39 & 0,737 & 0,794 & 0,928 \\
\hline Média & 0,903 & 0,955 & 0,946 \\
\hline Desvio padrão & 0,129 & 0,092 & 0,095 \\
\hline
\end{tabular}

relevância do entorno no desempenho das unidades. Em (13) também percebe-se a queda do desvio padrão, a diminuição das diferenças de eficiência e da heterogeneidade das DMUs, o que confirma a correção do efeito do entorno.

0 modelo (13) fornece, ainda, as melhorias radiais, conforme a Tabela 2. Elas podem orientar e induzir a discussão sobre ações que permitam reduzir os custos atuais e determinar metas a serem atingidas. Observa-se que a economia poderia ser de, no mínimo: 47 funcionários, 62 professores e $4.151 \mathrm{~m}^{2}$ em área construída. Embora haja um número alto de funcionários e professores não efetivos na UEG, acredita-se que as decisões para elevar a eficiência da instituição devam ser orientadas à maximização
Tabela 2. Melhorias radiais para a eficiência - BCC-10 (13).

\begin{tabular}{cccr}
\hline & \multicolumn{3}{c}{ Melhora (redução radial) } \\
\cline { 2 - 4 } & $\mathbf{l}_{1}$ & $\mathrm{l}_{2}$ & \multicolumn{1}{c}{$\mathrm{l}_{3}$} \\
\hline 6 & 1,58 & 3,35 & 128,32 \\
13 & 8,75 & 11,90 & $1.082,90$ \\
14 & 1,99 & 3,77 & 193,64 \\
16 & 1,55 & 1,93 & 84,17 \\
19 & 6,69 & 7,14 & 481,45 \\
21 & 5,76 & 11,26 & 632,76 \\
22 & 6,22 & 5,33 & 479,29 \\
34 & 1,47 & 3,26 & 126,34 \\
37 & 6,48 & 6,48 & 409,40 \\
38 & 0,04 & 0,05 & 4,25 \\
39 & 6,39 & 7,21 & 528,23 \\
$\Sigma$ & 47 & 62 & 4.151 \\
\hline
\end{tabular}

Tabela 3. Melhorias radiais para a eficiência - BCC-00 (14).

\begin{tabular}{|c|c|c|c|c|c|c|}
\hline \multirow{2}{*}{ DMU } & \multirow{2}{*}{ Score $\omega$} & \multicolumn{5}{|c|}{ Melhora (aumento radial) } \\
\hline & & $0_{1}$ & $\mathrm{O}_{2}$ & $\mathrm{O}_{3}$ & $\mathrm{O}_{4}$ & $\mathrm{O}_{5}$ \\
\hline 6 & 1,05 & 8,2 & 8,2 & 2,0 & 0,5 & $1.432,1$ \\
\hline 13 & 1,49 & 12,4 & 5,0 & 2,5 & 0,5 & $1.095,4$ \\
\hline 14 & 1,15 & 12,9 & 4,3 & 0,1 & 0,1 & 721,1 \\
\hline 16 & 1,18 & 0,2 & 4,4 & 2,2 & 0,2 & $1.017,0$ \\
\hline 19 & 1,46 & 2,5 & 4,9 & 2,5 & 0,5 & $1.142,0$ \\
\hline 21 & 1,31 & 2,3 & 6,9 & 2,3 & 0,3 & $1.017,4$ \\
\hline 22 & 1,55 & 0,5 & 5,1 & 5,1 & 0,5 & $1.249,7$ \\
\hline 34 & 1,07 & 0,1 & 8,3 & 2,1 & 0,1 & $1.265,0$ \\
\hline 37 & 1,41 & 0,4 & 4,8 & 4,8 & 0,4 & 972,9 \\
\hline 38 & 1,01 & 0,0 & 0,0 & 6,0 & 0,0 & 365,8 \\
\hline 39 & 1,23 & 0,2 & 6,7 & 2,2 & 0,2 & $1.412,7$ \\
\hline & $\Sigma$ & 40 & 59 & 32 & 3 & 11691 \\
\hline
\end{tabular}

dos produtos, a partir dos recursos disponíveis, considerando a demanda reprimida por ensino superior gratuito no estado.

A execução do modelo BCC-00 (14) resulta na informação que determina a maximização da produção a partir dos insumos disponíveis ou quando os insumos, por questões políticas ou práticas, não devem ser reduzidos. Nota-se, na Tabela 3 , que as 11 DMUs ineficientes são as mesmas do modelo anterior. A média dos índices agora é 1,07, o que indica que a eficiência, dadas as condições do entorno, pode ser incrementada em 7\%, no mínimo. As melhorias radiais poderão aumentar o número de alunos matriculados em 11.691, os cursos de graduação em 59, os projetos de pesquisa em 40, os cursos de qualificação profissional em 32 e os cursos de pós-graduação em 3.

Além disso, combinando-se BCC-OO (14) e CCR-00 (8) pode-se decompor o índice de eficiência global em termos de eficiência pura e de escala. Conforme já explicado, se $\mathrm{EE}=1$, então $\mathrm{ET}_{\mathrm{CR}}=\mathrm{ET}_{\mathrm{VR}}$, o que indica que a DMU não apresenta ineficiência de escala e, assim, opera com um porte ótimo. Se a DMU apresenta ineficiência de escala (EE $>1)$, pode-se utilizar o somatório dos $\lambda_{\mathrm{j}}$ do modelo CCR-00 (8) 
Tabela 4. Ineficiência global, pura, de escala, tipo de rendimento e DMUs benchmarks.

\begin{tabular}{cccccl}
\hline DMU & $\begin{array}{c}\mathrm{ET}_{\mathrm{CR}} \\
\mathrm{CCR}-00 \text { (8) }\end{array}$ & $\begin{array}{c}\mathrm{ET}_{\mathrm{VR}} \\
\mathrm{BCC}-00(14)\end{array}$ & $\begin{array}{c}\mathrm{EE}= \\
\mathrm{ET}_{\mathrm{CR}} / \mathrm{ET}_{\mathrm{VR}}\end{array}$ & $\begin{array}{c}\text { Tipo de } \\
\text { rendimento }\end{array}$ & $\begin{array}{c}\text { DMUs } \\
\text { benchmarks }\end{array}$ \\
\hline 4 & 1,46 & 1,00 & 1,456 & Crescente & $10,15,35$ \\
6 & 1,28 & 1,05 & 1,226 & Crescente & $2,10,25,27,30,35$ \\
14 & 1,17 & 1,15 & 1,025 & Crescente & $2,18,27$ \\
16 & 1,29 & 1,18 & 1,099 & Crescente & $10,18,30,35,36$ \\
19 & 1,80 & 1,46 & 1,233 & Crescente & $10,17,32,35$ \\
21 & 1,34 & 1,31 & 1,020 & Crescente & $10,17,27,32$ \\
28 & 2,37 & 1,00 & 2,371 & Crescente & $7,26,27,35$ \\
33 & 1,24 & 1,00 & 1,241 & Crescente & $7,10,27,31,35$ \\
39 & 1,28 & 1,23 & 1,039 & Crescente & $10,17,32,35$ \\
3 & 1,12 & 1,00 & 1,117 & Decrescente & $7,31,36$ \\
5 & 1,02 & 1,00 & 1,023 & Decrescente & $7,10,35,36$ \\
11 & 1,38 & 1,00 & 1,376 & Decrescente & $1,2,9,35$ \\
13 & 1,52 & 1,49 & 1,019 & Decrescente & $2,10,27,35$ \\
20 & 1,70 & 1,00 & Decrescente & $7,26,31,36$ \\
22 & 1,56 & 1,55 & 1,697 & Decrescente & $10,17,27,35$ \\
24 & 1,59 & 1,00 & 1,013 & Decrescente & $7,8,26,27$ \\
29 & 1,10 & 1,00 & Decrescente & $9,30,35,36$ \\
34 & 1,08 & 1,07 & 1,095 & Decrescente & $10,17,30,32$ \\
37 & 1,56 & 1,41 & 1,005 & Decrescente & $10,18,30,35,36$ \\
38 & 1,03 & 1,01 & Decrescente & $7,8,12,26$ \\
\hline
\end{tabular}

para determinar o tipo de rendimento. Os resultados desses procedimentos, conforme mostra a Tabela 4, indicam que 20 DMUs têm ineficiência de escala e somente 19 estão operando com o tamanho ótimo e a máxima produtividade. Dessas 20 unidades: a) nove apresentam eficiência pura, ou seja, sua ineficiência é decorrente, exclusivamente, da inadequada escala de produção, e b) 11 estão subdimensionadas e nove, superdimensionadas.

Essa informação poderia ser utilizada como subsídio para definir políticas de expansão e remanejamento de recursos. Por exemplo, nas 11 DMUs com rendimentos decrescentes, recomendam-se as melhorias do modelo CCR-10 (7). Essa ação reduz o porte. Nas nove DMUs com rendimentos crescentes, aconselham-se as melhorias do modelo CCR-00 (8) e o investimento ou remanejamento dos recursos das DMUs com rendimentos decrescentes, já que assim podem aproveitar os retornos crescentes. A solução da ineficiência pura e de escala poderia trazer uma redução de custos associada a uma expansão da produção. As melhoras radiais do modelo CCR-00 (8) sugerem um aumento de 20,21\% dos outputs.

Por último, o modelo CCR-00 (8) fornece o conjunto de DMUs benchmarks em que as ineficientes podem espelhar-se para melhorar seu desempenho. Aquela que participou com maior frequência é chamada de Global Leader, sendo, neste caso, a unidade 35. Porém, antes de tirar qualquer conclusão é recomendável a realização de auditorias internas para identificar as fontes dessa superioridade. Deve-se, nesse sentido, verificar se os produtos estão sendo fornecidos segundo os padrões mínimos de qualidade e se os insumos não estão sendo superutilizados.

\section{Conclusões}

A identificação da relevância do contexto, a correta gestão dos recursos e a maximização da produção universitária são prioridades a serem perseguidas pelo ensino superior público. Este trabalho teve como objetivo analisar essas prioridades e mostrar a contribuição do DEA na avaliação da eficiência das unidades da UEG, prestando especial atenção ao contexto socioeconômico diferenciado nos quais elas operam.

Para aplicar o método proposto, utilizaram-se os dados disponíveis. Embora a contribuição desses dados com a análise não seja exaustiva, os resultados obtidos permitem à UEG fazer uma importante reflexão das condições existentes.

0 modelo BCC-E-10 (10), calculado sem considerar o entorno socioeconômico, mostra que existem 20 DMUs ineficientes. A média dos índices é 90\%, dos quais aproximadamente 5\% sãoatribuídos à ineficiência de gestão e 5\% ao impacto negativo do ambiente. Neste sentido, todas as unidades ineficientes sofrem efeito do contexto socioeconômico. Corrigindo-se esse efeito, conforme modelo BCC-10 (13), cai o número de ineficientes para 11. A média dos índices passa para 95\%, o que indica que a UEG, descontado o impacto do entorno, poderia produzir o mesmo reduzindo os insumos em 5\%, no mínimo. Alternativamente, a UEG, conforme modelo BCC-OO (14), poderia incrementar sua produção em 7\% mantendo o nível atual de insumos.

A pesquisa mostrou, também, que 11 DMUs estão subdimensionadas e nove, superdimensionadas. Essa informação poderia ser utilizada para definir políticas 
de redução de custos associadas a uma expansão que otimize o potencial de crescimento e o porte ideal das unidades.

Para finalizar, é importante ressaltar que a análise e as sugestões decorrentes deste trabalho estão condicionadas às DMUs e às variáveis contempladas bem como ao princípio de que todos os demais fatores envolvidos são idênticos. Por ser a eficiência uma medida relativa, qualquer unidade e variável acrescentada ou excluída da análise poderá modificar os resultados. Nesse sentido, como linha de investigação futura, recomenda-se reaplicar os modelos após uma profunda discussão na UEG que defina as variáveis que melhor contribuam com a análise da eficiência. Dessa forma, o DEA pode transformar-se numa valiosa ferramenta de avaliação que permitirá orientar a discussão do contínuo processo de planejamento, organização e controle do trabalho para alcançar, eficientemente, os objetivos da universidade.

\section{Referências}

ANGULO-MEZA, L.; SANTOS, F. R. Modelos DEA com variáveis não controláveis na avaliação de veículos do segmento B. Sistemas \& Gestão, v. 2, n. 3, 2007.

BANKER, R.; CHARNES, A.; COOPER, W. Some models for estimating technical scale inefficiencies in Data Envelopment Analysis. Management Science, v. 30, n. 9, 1984. http://dx.doi.org/10.1287/mnsc.30.9.1078

BANKER, R. D.; MOREY, R. C. Efficiency analysis for exogenously fixed inputs and outputs. Operations Research, v. 34, n. 4, p. 513-521, 1986. http://dx.doi. org/10.1287/opre.34.4.513

CHARNES, A.; COOPER, W.; RHODES, E. Measuring the efficiency on decision marking units. European Journal of Operational Research, v. 2, n. 6, 1978. http://dx.doi. org/10.1016/0377-2217(78)90138-8

COLEMAN, J. S. et al. Equality of educational opportunity. Washington: U.S. Government Printing Office, 1966.

COLL, V.; BLASCO, 0. Evaluación de la eficiencia mediante el análisis envolvente de datos. Introducción a los modelos básicos. Valencia: Universidad de Valencia, 2006.
Disponível em: <http://www.eumed.net/>. Acesso em 22 set. 2008.

COOPER, W.; SEIFORD, L. M.; TONE, K. Data envelopment analysis: a comprehensive text with model, applications, references and DEA-solver software. Boston: Kluwer Academic Publishers, 2007.

DEBREU, G. The coefficient of resource utilization. Econometrica, v. 19, n. 3, 1951. http://dx.doi. org/10.2307/1906814

FARRELL, M. J. The measurement of productive efficiency. Journal of the Royal Statistical Society, Series $A$, v. 120, n. 3, p. 253-290, 1957. http://dx.doi. org/10.2307/2343100

HANUSHEK, E. The economics of schooling: Production and Efficiency in Public Schools. Journal of Economic Literature, v. 24, n. 3, p. 1141-1177, 1986.

KOOPMANS, T. C. An analysis of production as an efficient combination of activities. In: KOOPMANS, T. C. (Ed.). Activity analysis of production and allocation, Cowles Commission for Research in Economics. New York: Wiley, 1951. Monograph n. 13.

LINS, M. P. E.; ANGULO-MEZA, L. (Eds.). Análise envoltória de dados e perspectivas de integração no ambiente de apoio à decisão. Rio de Janeiro: COPPE/UFRJ, 2000.

LOZANO-VIVAS, A.; PASTOR, J. T. E.; PASTOR, J. M. An efficiency comparison of european banking systems operating under different environmental conditions. Journal of Productivity Analysis, v. 18, n. 1, p. 59-77, 2002. http://dx.doi.org/10.1023/A:1015704510270

MUÑIZ, M. Introducción de variables de control en modelos DEA. In: ÁlVAREZ, P. (Ed.). La Medición de la eficiencia y la productividad. Madrid: Ed Pirámide, 2001.

PALOMARES-MONTERO, D.; GARCÍA-ARACIL, A.; CASTROMARTÍNEZ, E. Evaluación de las instituciones de educación superior: revisión bibliográfica de sistema de indicadores. Revista Española de Documentación Científica, v. 31, n. 2, 2008.

SOUZA, G. S. et al. Economic efficiency of Embrapa's research centers and the influence of contextual variables. Pesquisa Operacional, v. 27, p. 15-26, 2007. http:// dx.doi.org/10.1590/S0101-74382007000100002

UNIVERSIDADE ESTADUAL DE GOIÁS. Relatório de gestão, dez. 2007. Disponível em: <http://www.dirplan.ueg.br>. Acesso em: 25 maio 2008.

VASCONCELlOS, M. S.; OLIVEIRA, R. G. Microeconomia. São Paulo: Atlas, 1996.

\title{
Efficiency and impact of the context in the management through the DEA: case study of the UEG
}

\begin{abstract}
This work shows the contribution of the method Data Envelopment Analysis (DEA) in the evaluation of the efficiency of organizations that operate in differentiated contexts. The DEA application in the State University of Goiás (UEG) demonstrates that 20 of the 39 analyzed units are inefficient. The 20 suffer negative effect from context measured by HDI-M. Isolating this effect, the number of inefficient units falls to 11 and the mean of the efficiency indexes passes to 0.95 which indicates that these units could produce the same outputs, reducing the inputs at least 5\%. Alternatively, UEG, keeping the current level of inputs, could increase its production by 7\%. This research has shown, also, that 11 units are too small and nine are too large. This information could be used to reduce costs and to optimize the size of the units.
\end{abstract}

\section{Keywords}

Data envelopment analysis. Institutional evaluation. Context. Efficiency. 